\title{
Soziale Teilhabe durch Arbeitsintegration
}

Arbeitsfeld Arbeitsintegration

Martin Stalder ${ }^{1}$, Karin Stoop ${ }^{2}$

Der nachfolgende Artikel beleuchtet das Thema der Arbeitsintegration suchtmittelabhängiger Menschen. Die Ziele der Arbeitsintegration sind breit gefächert. Beginnend bei der sozialen Integration im Rahmen von Beschäftigungsangeboten von sozialen Einrichtungen bis hin zur Vermittlung in Arbeitsstellen im ersten Arbeitsmarkt unterstützen Sozialarbeitende ihre Klientinnen und Klienten.

\section{Arbeitsfeld}

Welche Rolle fällt der Sozialen Arbeit in der Arbeitsintegration zu, welchen Teil übernimmt die Medizin, und welche weiteren Rahmenbedingungen müssen erfüllt sein, damit als erster Schritt zumindest die Teilnahme an Beschäftigungsprojekten gelingen kann? Was braucht es, um private Arbeitgeber als Partner gewinnen zu können?

Erwerbsarbeit sichert in erster Linie die ökonomische Unabhängigkeit. Sie dient aber auch der gesellschaftlichen Integration. Arbeit schafft Identität und Zugehörigkeit. Besteht kein Zugang zu einer Erwerbsarbeit, fällt meist nicht nur die finanzielle Autonomie weg, sondern auch die Teilhabe an einem wichtigen Teil der Gesellschaft.

Fehlt die Erwerbsarbeit oder fehlen Leistungen einer Sozialversicherung, greift die gesetzliche Sozialhilfe. Diese sichert die finanzielle Existenz bedürftiger Personen, fördert die wirtschaftliche und persönliche Selbständigkeit und gewährleistet die soziale und berufliche Integration. ${ }^{3}$

\footnotetext{
${ }^{1}$ Sozialarbeiter FH, Soziale Dienste Stadt Solothurn.

2 Sozialarbeiterin FH, Geschäftsleiterin, PERSPEKTIVE Region Solothurn-Grenchen.

${ }^{3}$ Auf diese Weise definiert die Schweizerische Konferenz für Sozialhilfe SKOS die Ziele der Sozialhilfe, vgl. www.tinyurl.com/yyts97ye, Zugriff 12.07.2019.
} 
Die berufliche Integration - hier Arbeitsintegration genannt - ist somit ein zentrales Aufgabengebiet der gesetzlichen Sozialhilfe. Durch die Integration in die Arbeitswelt kann die finanzielle Autonomie der Sozialhilfeempfängerinnen und Sozialhilfeempfänger wieder erreicht werden. Davon ausgenommen sind die Personen, welche durch den Zugang zu Leistungen einer Sozialversicherung (z. B. IV) keine Leistungen der gesetzlichen Sozialhilfe mehr benötigen.

\subsection{Ziel und Zweck der Arbeitsintegration}

Mit Hilfe arbeitsmarktlicher Massnahmen soll den Sozialhilfebezügerinnen und -bezügern ein Wiedereinstieg oder teilweise sogar ein erster Einstieg in die Arbeitswelt ermöglicht werden. Eine berufliche Qualifizierung ist hierbei oft das zentrale Element.

Wenn suchtkranke, stellenlose Menschen nach dem Ausschöpfen der Rahmenfrist der Arbeitslosenversicherung ihre Existenz durch Leistungen der Sozialhilfe bestreiten müssen, liegt das Problem aber nicht nur an einer fehlenden Qualifikation oder an einem Mangel an verfügbaren Arbeitsplätzen. Vielmehr können diese Menschen zum aktuellen Zeitpunkt die von der Wirtschaft definierten Anforderungen an eine Arbeitskraft nicht erfüllen. Ihre gesundheitlichen Einschränkungen oder ihre durch die Sucht bedingte Instabilität in der Leistungserbringung und die teilweise fehlende Zuverlässigkeit verunmöglichen die Einhaltung der Pflichten eines Arbeitnehmers/einer Arbeitnehmerin in einem regulären Arbeitsverhältnis.

Für diese Gruppe muss deshalb, zumindest für eine bestimmte Zeit, vor der beruflichen Integration eine soziale Integration im Fokus stehen.

Der Begriff soziale Integration umfasst vieles. Es geht dabei um Teilhabe am gesellschaftlichen Leben. Durch gezielte Integrationsmassnahmen soll eine drohende Desintegration von Suchtkranken eingedämmt werden (SKOS Richtlinien D.2) ${ }^{4}$.

Für akut suchtkranke Menschen, welche sich in Beschäftigungsprojekten der ambulanten Suchthilfe in einer Tagesstruktur befinden, ist die berufliche Integration auf die Schnelle meist unrealistisch. Suchtkranke

\footnotetext{
${ }^{4}$ Vgl. www.tinyurl.com/yxfxxmd3, Zugriff 5.11.2019.
} 
Menschen stehen als Folge ihrer Krankheit und der damit verbundenen Stigmatisierung am Rande unserer Gesellschaft.

\subsection{Zielgruppen im Arbeitsfeld}

Gemäss act-info Jahresbericht 2016 (Delgrande Jordan 2017) sind bei einem Eintritt in eine Suchtbehandlung 32,8 \% der KlientInnen in einer Festanstellung und 6,5\% in einer Teilzeitanstellung. Bei diesen $39 \%$ der KlientInnen, die noch in einem Arbeitsverhältnis stehen, muss der Erhalt des Arbeitsplatzes ein zentrales Ziel der Unterstützung sein. Auf diesen Aspekt wird im vorliegenden Artikel nicht weiter eingegangen.

Bezogen auf die genannte Statistik verfügen mehr als $60 \%$ der von Sucht betroffenen Menschen, die in eine ambulante Suchtbehandlung einsteigen, nicht über ein regelmässiges Erwerbseinkommen. Der Lebensunterhalt wird vorwiegend durch Sozialhilfe oder einer Rentenleistung bestritten.

Der geringere Teil dieser Menschen ist durch eine Rentenleistung der IV und AHV keine prioritäre Zielgruppe für Arbeitsintegrations- respektive Beschäftigungsmassnahmen.

\section{Arbeitsintegration in der PERSPEKTIVE Region Solothurn-Gren- chen}

Die PERSPEKTIVE Region Solothurn-Grenchen bietet ein umfassendes Angebot in der ambulanten Suchtbehandlung. Die Dienstleistungen umfassen: Prävention, Beratung, Wohnen, Arbeit, Gassenküche und Kontaktund Anlaufstelle. Basierend auf dem kantonalen Sozialgesetz sind die Gemeinden für die Finanzierung dieser Angebote zuständig. Die Leistungen werden in einem Leistungskatalog definiert und sind gemäss diesen Vorgaben umzusetzen.

Im Bereich Arbeit wird mit einem stufengerechten Aufbau eine leistungsabhängige Beschäftigung ermöglicht.

Bei den ausgeführten Arbeiten handelt es sich um diverse Hilfsarbeiten wie Räumungen, Umzüge, Reinigungen, Gartenarbeiten und Littering-Aufträge. Sie erfolgen mehrheitlich im Auftrag von Privatpersonen, 
teilweise aber auch von der öffentlichen Hand. Die Betreuung der Klientinnen und Klienten während der Arbeiten erfolgt durch Vorarbeiter, welche teilweise über eine Ausbildung als Arbeitsagogen verfügen. Die sozialarbeiterische Betreuung wird durch die Case Managerinnen und Case Manager gewährleistet.

\subsection{Angebotspalette der PERSPEKTIVE}

Die drei Programme Gemeindearbeitsplätze (GAP), Tagesbeschäftigung und Gartenbau stellen unterschiedliche Anforderungen an die Teilnehmenden und sind auch mit unterschiedlichen Zielsetzungen verbunden.

Die Teilnahme am GAP hat eine regelmässige Tagesstruktur zum Ziel. Die Teilnehmenden sollten, wenn möglich, an fünf Tagen die Woche jeweils einen halben Tag im Programm erscheinen und leichtere Aufgaben ausführen. Diese stellen keine grossen körperlichen Anforderungen (z. B. Unterhaltsarbeiten in der Gemeinde). In der Tagesbeschäftigung dauern die Arbeitstage in der Regel bis acht Stunden. Auch sind die Aufgaben körperlich belastender (Hausräumungen und Zügelarbeiten, Gärtnerarbeiten, Holzarbeiten). Für die Teilnahme am GAP oder an der Tagesbeschäftigung muss eine Kostengutsprache der Sozialhilfe vorliegen.

Der Gartenbau ist ein Teillohnprogramm. Die Teilnehmenden müssen eine hohe Zuverlässigkeit bei der Präsenz an den Tag legen. Das Teillohnprojekt ist für die zuweisenden Stellen kostenlos. Pro geleistete Arbeitsstunde wird den Projekteilnehmenden einen Nettostundenlohn von Fr 8.00 ausbezahlt. Dies ermöglicht es ihnen einen Teil des Lebensunterhaltes selber zu finanzieren.

In keinem der Programme kann eine neue Rahmenfrist der Arbeitslosenversicherung generiert werden. Die Vermittlung in den ersten Arbeitsmarkt ist kein explizites Ziel. KlientInnen, die aufgrund ihrer Leistung dazu fähig sind, werden in andere dafür ausgerichtete Projekte im Kanton vermittelt.

Während der Teilnahme in unseren Programmen werden alle KlientInnen durch einen Case Manager oder eine Case Managerin betreut. Diese Fachpersonen aus dem Berufsfeld der Sozialen Arbeit sind verantwortlich dafür, dass regelmässig mit allen am Prozess Beteiligten (z. B. Zuweiser, 
behandelnde ÄrztInnen, Fachpersonen der Beratung, Wohnbegleitung oder Bewährungshilfe, Angehörige etc.) ein Austausch stattfindet und bei Bedarf neue Unterstützungsleistungen erschlossen werden.

Die Arbeitsangebote der PERSPEKTIVE sind vom Amt für soziale Sicherheit des Kantons Solothurn akkreditiert. Der Kanton hatte 2016 mittels Angebotsplanung den gesamten Dienstleistungsmarkt der Arbeitsintegration nach einer umfassenden Überprüfung neu reguliert. Es können neu nur noch Programmkosten von akkreditieren Angeboten durch die Sozialhilfe finanziert und über den kantonalen Lastenausgleich abgerechnet werden. Die Angebotsplanung regelt zudem die Anzahl Plätze in den unterschiedlichen Angeboten von Beschäftigung, Qualifizierung und Coaching.

\subsection{Inhaltliche Ausgestaltung der Angebote}

Die Teilnahme an einem unserer Beschäftigungsprogramme soll für die Klientinnen und Klienten sinnstiftend sein. Sinnstiftend nicht nur hinsichtlich der Tagesstruktur oder der möglichen sozialen Kontakte, sondern auch bezogen auf eine erfüllende Beschäftigung oder Arbeit. Mit Angeboten, die Tätigkeiten in arbeitsmarktähnlichen Arbeitsfeldern insbesondere im handwerklichen oder industriellen Bereich beinhalten, kann dies ermöglicht werden. Die Mehrheit der Klienten ist männlich, was sich auch auf die Ausrichtung der Arbeitsfelder auswirkt.

Klassische weibliche Tätigkeitsfelder wie z. B. Pflege eignen sich bei akut suchtkranken Menschen weniger.

Bei Arbeiten im handwerklichen Bereich ist gut ersichtlich, was geleistet wurde. Wichtig ist, dass die Programmteilnehmenden in ihrem Alltag etwas tun können, das einen Wert darstellt, resp. dass die geleistete Arbeit einen Nutzen für Dritte hat. Sie erledigen einen Auftrag, der von der Kundschaft real nachgefragt wird.

Die Auswahl der konkreten Projekte in den einzelnen Programmen muss aber sorgfältig getroffen werden, damit bei der Auftragsakquisition nicht der Vorwurf der Konkurrenzierung des Gewerbes aufkommt.

Die Beschäftigung oder Arbeit soll mit Verantwortungsübernahme verbunden sein. Die Klientel soll sich bei der Ausführung der Tätigkeit 
weder unter- noch überfordert fühlen. Eine nahe Begleitung durch die ArbeitsagogInnen erlaubt es, die Arbeiten den Kompetenzen anzupassen und so die für eine positive Entwicklung notwendigen Erfolgserlebnisse zu ermöglichen. Positive Erfahrungen sind Treiber in der persönlichen Weiterentwicklung und für den weiteren Prozessverlauf.

\section{Funktion und Qualifikation der Sozialen Arbeit im Arbeitsfeld}

\subsection{Funktion und Stellenwert der Sozialen Arbeit}

Die Sozialgesetzgebung, die Möglichkeiten der wirtschaftlichen Sozialhilfe verbunden mit weiteren finanziellen Mitteln, sind die Grundlage, um Ressourcen für die Arbeitsintegration zu erschliessen. Die Soziale Arbeit unterstützt mit gezielten Massnahmen wie Beratung, Finanzierung von Projekteinsätzen oder Weiterbildungen suchtkranke Menschen in der sozialen Integration. Dafür orientiert sie sich an den gegebenen gesetzlichen Rahmenbedingungen der Sozialhilfe sowie des Arbeitsrechts.

Soziale Arbeit und damit auch Soziale Arbeit im Kontext der Arbeitsintegration nehmen potentiell die gesamte Lebensführung ihrer Klientel in den Blick und wirken damit in der gesamten Lebenssituation ihrer Klientel. Die Komplexität des Alltags und die Wechselwirkung der verschiedenen Lebensbereiche machen es unabdingbar, dass die Fachpersonen in der Sozialen Arbeit mit den KlientInnen deren ganze Lebenssituation fundiert betrachten und darauf aufbauend Schritte der Veränderung und Unterstützung vornehmen. Eine sorgfältige Anamnese ist eine wichtige Grundlage für die anstehende Veränderung und Basis für alle Interventionen. Sozialarbeitende erschliessen notwendige Ressourcen und stossen mit Beratung Veränderungsprozesse an.

Die oft mehrschichtige Problemlage der betroffenen KlientInnen bedarf eines Helfernetzwerks unterschiedlicher Professionen. Im Sinne einer effektiven und auch effizienten Dienstleistungserbringung ist es wichtig, dass sich die Akteure austauschen und eine gemeinsame Zielrichtung anstreben. Das Case Management bildet hier das wesentliche methodische Element. Wenn klar ist, wer für den Informationsaustausch innerhalb des 
Helfernetzes verantwortlich ist und welche Informationen im Einverständnis der Klientinnen und Klienten ausgetauscht werden können, kann gemeinsam an einem Strick gezogen oder eben ein Prozess angestossen werden.

Kritisch anzumerken gilt, dass im klassischen Feld der Sozialen Arbeit immer weniger Fachpersonen tätig sind, die über Berufserfahrung in handwerklichen oder gewerblichen Tätigkeiten verfügen. Dies ist eine Folge der aktuellen Bildungslandschaft und für die Soziale Arbeit in der Arbeitsintegration sicher kein Vorteil. Dazu kommen genderspezifische Umstände: Soziale Arbeit ist ein typischer Frauenberuf mit entsprechender beruflicher Sozialisation, während die Zielgruppe der Arbeitsintegration mehrheitlich männlich ist.

\subsection{Weiterbildung}

Die Thematik der Arbeitsintegration resp. des Eingliederungsmanagements wird in Fachkursen, Fachtagungen oder im Rahmen eines CAS/DAS an verschiedenen Fachhochschulen und vom Dachverband der sozialen und beruflichen Integration 5 angeboten .

Diese Lehrgänge berufen sich auf vielseitige Methoden, Verfahren und Instrumente für zielgruppenspezifische Bereiche. Die Diversität der Angebote widerspiegelt die fachliche Breite der Arbeitsintegration. Insbesondere die Fachhochschulen entwickeln laufend weitere Lehrgänge in diesem Bereich.

\section{Kooperation im Arbeitsfeld}

Wie eingangs erwähnt, ist die Lebenssituation der Teilnehmenden in Arbeitsintegrationsprojekten oft durch diverse Probleme belastet. Es ist deshalb zentral, dass sich fallführende Sozialarbeiterinnen und Sozialarbeiter mit Fachstellen vernetzen und so ein unterstützendes Helfernetz aufbauen. Zentral erachten wir die Zusammenarbeit mit ÄrztInnen, in der Re-

\footnotetext{
${ }^{5}$ www.arbeitsintegrationschweiz.ch
} 
gel sind dies die HausärztInnen, und mit ArbeitsagogInnen. Die HausärztInnen sind für die Festlegung der Arbeitsfähigkeit verantwortlich. Die ArbeitsagogInnen ihrerseits sind die Fachleute, die die KlientInnen im Alltag ganz konkret befähigen, sich aktiv an einem Arbeitsprozess zu beteiligen.

\subsection{Zusammenarbeit mit HausärztInnen}

Die HausärztInnen sind sehr wichtige PartnerInnen in der interprofessionellen Zusammenarbeit bezogen auf die Arbeitsintegration von suchtkranken Menschen. Den ÄrztInnen ist es vorbehalten, die Arbeitsfähigkeit medizinisch zu bescheinigen, wobei diese aber in der Regel in Bezug auf den ersten Arbeitsmarkt beurteilt wird. Eine eingeschränkte Arbeitsfähigkeit im ersten Arbeitsmarkt ist jedoch nicht gleichzusetzen mit einer eingeschränkten Arbeitsfähigkeit im zweiten Arbeitsmarkt.

Entsprechend notwendig ist es, dass die Verantwortlichen der Sozialen Arbeit gegenüber ÄrztInnen darlegen, welche Anforderungen in den Arbeitsintegrationsprojekten an die Teilnehmenden gestellt werden. Die ÄrztInnen müssen genau wissen, welche physischen und psychischen Belastungen mit der Beschäftigung verbunden sind, damit sie die Beschäftigungsfähigkeit korrekt einschätzen können.

\subsection{Einbezug der Arbeitsagoginnen und Arbeitsagogen}

ArbeitsagogInnen sind dafür besorgt, dass Menschen mit einer Suchtmittelabhängigkeit innerhalb der jeweiligen Arbeitsintegrationsprojekte praxisnah und unmittelbar gefördert werden. Neue Kompetenzen sollen schrittweise eingeübt und erlangt werden.

ArbeitsagogInnen vermitteln ganz praxisnah verhaltensbezogene und fachliche Kompetenzen in den jeweiligen Arbeitsbereichen. Damit bieten sie wichtige Unterstützung bei der Reintegration der KlientInnen in den Arbeitsmarkt.

Arbeitsagogik wird als Zweitberuf erlernt. Alle AkteurInnen in diesem Berufsfeld verfügen über einen Herkunftsberuf und kennen damit die Anforderungen der Wirtschaft und des Gewerbes an die Arbeitnehmenden aus eigener Erfahrung sehr gut. Wir erachten diese Kompetenz als zentral für die Tätigkeit in der Arbeitsintegration. Damit wird eine grosse 
Nähe zum Berufsalltag geschaffen und eine gewisse Normalität vermitteln. Ein Klient beschrieb dies treffend: «Mit meinem Vorarbeiter (Arbeitsagoge) kann ich in einer normalen Sprache reden».

Durch die räumliche Nähe und das gemeinsame Arbeiten teilen ArbeitsagogInnen und Klienten und Klientinnen den (Arbeits-)Alltag. Sie erleben die Fortschritte, aber auch die auftretenden Schwierigkeiten unmittelbar. Dieses Setting ist ein zentrales Element der Prozessgestaltung, d. h. Zielsetzungen können zeitnah und direkt besprochen und umgesetzt werden. Die Beraterinnen und Berater, das Case Management oder andere Fachstellen wie Berufsberatung oder Soziale Dienste haben hingegen nur punktuell Kontakt zu den Klienten und Klientinnen.

\section{Strukturelle Aspekte}

\subsection{Gesetzliche Vorgaben}

Viele Kantone in der Schweiz halten sich an die Richtlinien der SKOS. Im Kanton Solothurn wurden diese im Sozialgesetz (SG) des Kantons Solothurns (SG vom 31.1.2007) verbindlich übernommen (\$152 SG). ${ }^{6}$ Regelungen in der dazugehörigen Verordnung $(\mathrm{SV})^{7}$ definieren, welche Abweichungen von den Richtlinien zulässig sind.

Integrationsmassnahmen sind ein Bestandteil des Prinzips von Leistung und Gegenleistung in der Sozialhilfe (§ 148 Abs. 2 SG). Diese Verpflichtung zur Erbringung einer Gegenleistung ist damit auch für suchtmittelabhängige Personen verbindlich.

Die Ausgestaltung einer solchen Gegenleistung liegt in der Zuständigkeit der fallführenden Sozialarbeitenden und berücksichtigt die vielschichtigen Lebensumstände der betroffenen Person. Es gilt abzuschätzen, inwieweit die suchtmittelabhängige Person trotz ihrer Erkrankung in der

\footnotetext{
${ }^{6}$ Sozialgesetz (SG) Kanton Solothurn vom 31.Januar 2007 (Stand 1. Januar 2013): www.tinyurl.com/y45vqw7c, Zugriff 5.11.2019.

7 Sozialverordnung (SV) Kanton Solothurn vom 29. Oktober 2007 (Stand 1. Januar 2019): www.tinyurl.com/yxkj2oso, Zugriff 5.11.2019.
} 
Lage ist, einer Beschäftigung nachzugehen oder welche anderen Massnahmen geeignet sind, um die negativen Folgen der Krankheit zu mildern und wenn immer möglich eine Behandlung aufzunehmen.

\subsection{Fehlende finanzielle Anreize}

Die Teilnahme an einer Integrationsmassnahme sollte auch einen finanziellen Anreiz beinhalten. Seit einer Revision der Sozialverordnung (SV) 2015 als Folge von politischen Vorstössen werden die Integrationsmassnahmen mit Beschäftigungscharakter im Kanton Solothurn nicht mehr finanziell belohnt. Man geht davon aus, dass Teilnehmende dieser Angebote die minimale gesetzliche Gegenleistung als Verpflichtung erbringen und ihnen deshalb kein finanzieller Anreiz zusteht. Den SozialarbeiterInnen wurde damit ein unterstützendes Arbeitsinstrument entzogen. Die Motivation muss nun ausschliesslich durch gute Beratungsarbeit und passende Beschäftigung hergestellt werden.

Nur noch Massnahmen zur Qualifikation werden mit einer Integrationszulage (IZU) honoriert. Diese gilt ausschliesslich bei Projekten, welche die Integration in den ersten Arbeitsmarkt als Zielsetzung haben.

Bei der Angebotsplanung der arbeitsmarktlichen Massnahmen wurde einem mehrstufigem Modell Rechnung getragen. Von niederschwelliger Beschäftigung hin zu Qualifizierungs- und Coachingangeboten ist eine progressive Entwicklung möglich.

Mittels der kantonalen Vorgaben einer zeitlichen Beschränkung und der Pflicht, die erreichten Ziele zu dokumentieren, soll einer langfristigen Platzierung in Beschäftigungsprojekten entgegengewirkt werden. Es bedarf allerdings eines sorgfältigen Umgangs mit dieser Befristung der Beschäftigung. Die ganze Arbeitsintegration und Beschäftigung sind aufgrund der komplexen Problemsituationen oft ein langfristiger Prozess. Es gilt deshalb für die Sozialarbeiterinnen und Sozialarbeiter, diese komplexen Problemsituationen so darzulegen, dass auch von politischer Seite das Verständnis für diese Prozesse wächst. 


\subsection{Möglichkeiten der Sozialversicherungen}

Für BezügerInnen von Leistungen einer Sozialversicherung gelten besondere gesetzliche Grundlagen in Bezug auf soziale Integration. Insbesondere geht man bei einer Berentung nicht von einer Tätigkeit im ersten Arbeitsmarkt aus, zumindest nicht von einer vollzeitlichen. Auch die dem Sozialversicherungsverfahren vorgelagerten Eingliederungsbemühungen sind nicht mit den Bemühungen der Sozialhilfe vergleichbar. Diese zielen auf eine Rückkehr oder auf einen Verbleib in der Erwerbsarbeit ab. Die beruflichen Massnahmen finden oftmals direkt im ersten Arbeitsmarkt statt.

Bei einer Berentung besteht für Betroffene ein gut ausgebautes Netz an Möglichkeiten im zweiten Arbeitsmarkt. Die Anforderungen an die Anbieter werden vom Bundesamt für Sozialversicherungen definiert. Auch die Finanzierung solcher Angebote ist geregelt.

Die Soziale Arbeit orientiert sich in diesem Betätigungsfeld am Regelwerk oder an den gesetzlichen Bestimmungen der Sozialversicherungen.

Sozialarbeitende können den Wiedereingliederungsprozess durch Beratungs- und Beziehungsarbeit begleiten und durch Kenntnisse der Möglichkeiten der Sozialversicherungsleistungen den Zugang zu Weiterbildungen und damit das Erlangen von neuen Qualifikationen erschliessen.

\section{Herausforderungen und Chancen}

\subsection{Wirtschaftliche Entwicklung, Einbindung des Gewerbes}

Nebst bestehenden Angeboten von Arbeitsintegrationsmassnahmen sind vermehrt individuelle Lösungen zur Beschäftigung oder zur Arbeitsintegration notwendig. Dabei sollten mutige Lösungsansätze im ersten Arbeitsmarkt gesucht werden. Eine Möglichkeit besteht darin, die KlientInnen als Gegenleistung zu einem Sozialhilfebezug direkt in Industrie und Gewerbe zu vermitteln, ohne dass sofort eine Lohnzahlung erwartet wird. Damit wäre es dem Gewerbe und der Wirtschaft möglich, solche Nischenarbeitsplätze zu schaffen resp. zu reaktivieren. 
Solche Beschäftigungseinsätze im ersten Arbeitsmarkt können einen Mehrwert auf beiden Seiten schaffen. Die betroffenen KlientInnen erleben ihre Arbeitskraft im realen Umfeld. Die Unternehmen können ihrerseits soziale Verantwortung übernehmen.

Eine Tätigkeit in einem solchen Umfeld, wie es z. B. ein Gewerbebetrieb bietet, kann der eigenen beruflichen Einschätzung der Betroffenen einen positiven Schub verleihen. In einer Gesellschaft, welche dadurch geprägt ist, dass Menschen eine Leistung in Form von Arbeit erbringen, ist eine solche Anerkennung elementar. Die in einem Einsatzbetrieb der realen Wirtschaft erlebbaren Möglichkeiten und Grenzen, d. h. die Einsicht der eigenen Situation, können klärend sein und als Motivation zur Änderung beitragen oder aber die Akzeptanz der Grenzen fördern.

Eine derartige Beschäftigung ist nur für einen Teil der suchtkranken Menschen umsetzbar, aber für die Menschen, die dazu in der Lage sind, ein äusserst wertvoller.

Solche Einsätze dürfen aber nie bestehende Arbeitsplätze in einem Betrieb gefährden. Auch müssen sie für den Klienten oder die Klientin mit klaren Zielsetzungen begleitet sein. Sie müssen von vornherein wissen, ob für sie die Möglichkeit besteht, im Anschluss eine Anstellung im entsprechenden Betrieb zu bekommen, oder aber, ob sich dieser Einsatz klar auf die Erarbeitung von spezifischen Fachkompetenzen beschränkt. Es gilt dabei zu verhindern, dass sich die vermittelten Personen als billige Arbeitskraft wahrnehmen.

\subsection{Herausforderungen für die Soziale Arbeit?}

Die Soziale Arbeit kann bei Lösungsansätzen der individuellen Integration in den Arbeitsmarkt unter Einbezug von Betrieben und Unternehmen eine tragende und vermittelnde Rolle übernehmen. Dabei geht es nicht um das blosse Zuweisen von SozialhilfeempfängerInnen in Einsatzbetriebe. Die Aufgabe beinhaltet neben der Betreuung der KlientInnen insbesondere die Unterstützung von Arbeitgebenden. Engagierte Arbeitgebende übernehmen Aufgaben, die über eine gewöhnliche Mitarbeiterführung hinausgehen. Eine Person mit eingeschränkter Leistungsfähigkeit in einem Team zu integrieren braucht Wille und Bereitschaft auch von den ArbeitskollegIn- 
nen. Gerade hier ist oft eine vorgängige Abklärung nötig. Die Sozialarbeitenden müssen deshalb diese Einsätze mit dem Betrieb gut vorbereiten und begleiten. Die intensive Prozessbegleitung soll auch sicherstellen, dass ein solcher Einsatz nicht zur reinen Gratisarbeit verkommt, sondern qualifizierend bleibt. Eine Voraussetzung für eine solche Begleitung sind ausreichende zeitliche Ressourcen für die fallführenden SozialarbeiterInnen.

Die sorgfältige Begleitung von vermittelten Personen zusammen mit einem Verständnis für die unternehmerischen Belange der Einsatzbetriebe kann mittelfristig zu einer gewünschten Integration führen. Unverzichtbar ist dabei eine gezielte Netzwerkarbeit. Dies bedingt kurze und unkomplizierte Kommunikationswege. Es soll zu keinem Zeitpunkt ausser Acht gelassen werden, dass ein Einsatzbetrieb nicht die Integration von Betroffenen als Kerngeschäft betreiben kann, aber sehr wohl eine soziale Verantwortung wahrnimmt.

Unsere Erfahrung zeigt, dass kleine und mittlere Unternehmen (KMU) und hier insbesondere kleine Gewerbeunternehmen sehr wohl bereit für eine Zusammenarbeit sind. Es braucht den Schritt der Sozialen Arbeit, auf diese Betriebe zuzugehen, die Kooperation zu suchen und im Sinne einer tragenden Partnerschaft die Prozesse unkompliziert und pragmatisch zu gestalten. Kooperation ist möglich, Kooperation baut auf Beziehungen auf und diese gilt es zu pflegen.

\subsection{Zwingende Kompetenzanforderung an das Klientel als Folge der Digitalisierung}

Die auseinandergehende Entwicklung von Ansprüchen des Arbeitsmarktes einerseits und Ressourcen der Zielgruppe andererseits ist offensichtlich. Die Arbeitswelt verändert sich, was die Qualifikation der Arbeitnehmenden betrifft, mit der Digitalisierung aller Bereiche sehr rasch - Stichwort «Industrie 4.0». Auf Seiten der Klientinnen und Klienten sind die dazu passende Qualifikationen oftmals kaum oder gar nicht vorhanden.

Beschäftigungsprojekte sollen dem Erwerb dieser Qualifikationen vermehrt Beachtung schenken. Entsprechende Weiterbildungen sollten auch für suchtkranke Projektteilnehmende zum Angebot gehören. Damit wird nicht nur die Chance auf ein Weiterkommen im Integrationsprozess 
erhöht, sondern auch die Attraktivität der Angebote aus Sicht der Klientinnen und Klienten.

Je höherschwelliger ein Beschäftigungsangebot ist, je näher dieses am ersten Arbeitsmarkt ist, umso mehr gehört neben dem (Wieder-)Erlernen der Arbeitsfähigkeit auch die Grundausbildung in gängigen Softwareanwendungendazu.

\section{Literatur}

Delgrande Jordan, M. (2017): Stationäre Behandlung der Alkoholabhängigkeit - Ergebnisse der KlientInnenbefragung act-info2016 - Deskriptive Statistik. Lausanne: Sucht Schweiz.

Open Access Dieses Kapitel wird unter der Creative Commons Namensnennung 4.0 International Lizenz (http://creativecommons.org/licenses/by/4.0/deed.de) veröffentlicht, welche die Nutzung, Vervielfältigung, Bearbeitung, Verbreitung und Wiedergabe in jeglichem Medium und Format erlaubt, sofern Sie den/die ursprünglichen Autor(en) und die Quelle ordnungsgemäß nennen, einen Link zur Creative Commons Lizenz beifügen und angeben, ob Änderungen vorgenommen wurden.

Die in diesem Kapitel enthaltenen Bilder und sonstiges Drittmaterial unterliegen ebenfalls der genannten Creative Commons Lizenz, sofern sich aus der Abbildungslegende nichts anderes ergibt. Sofern das betreffende Material nicht unter der genannten Creative Commons Lizenz steht und die betreffende Handlung nicht nach gesetzlichen Vorschriften erlaubt ist, ist für die oben aufgeführten Weiterverwendungen des Materials die Einwilligung des jeweiligen Rechteinhabers einzuholen.

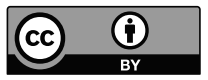

